\title{
Pancreatitis in a high HIV prevalence environment
}

\author{
F Anderson, ${ }^{1}$ FCS, MMed; S R Thomson, ${ }^{2}$ ChM, FRCS (Ed \& Eng) \\ ${ }^{1}$ Department of Surgery, Inkosi Albert Luthuli Central Hospital and School of Clinical Medicine, College of Health Sciences, Nelson R Mandela \\ School of Medicine, University of KwaZulu-Natal, Durban, South Africa \\ ${ }^{2}$ Department of Gastroenterology, Groote Schuur Hospital and Faculty of Health sciences, University of Cape Town, South Africa
}

Corresponding author: F Anderson (andersonf1@ukzn.ac.za)

\begin{abstract}
Background. Acute pancreatitis is common in HIV-positive individuals in reports from regions with a low incidence of HIV infection. This association has not been reported in areas with a high incidence of HIV infection.

Objective. To examine the prevalence and outcomes of HIV-associated acute pancreatitis in a high HIV prevalence environment, and trends over the period May 2001 - November 2010.

Methods. The records of patients admitted with acute pancreatitis from 2001 to 2010 were reviewed, looking for HIV status, CD4 counts and medications at presentation. The Glasgow criteria, organ failure, local complications and mortality were assessed.

Results. One hundred and six (16.9\%) of 627 patients admitted with acute pancreatitis during the study period were infected with HIV. Most were female (65.1\%) and black African (91.5\%). The serum amylase level was used to confirm acute pancreatitis in 50 patients, with a mean of $1569 \mathrm{IU} / \mathrm{L}$ (range 375 - 5 769), and urinary amylase in 56 patients, with a mean of $4083 \mathrm{IU} / \mathrm{L}$ (range 934 - 36 856). Alcohol was a less frequent cause of pancreatitis in the HIV-positive group than in patients who were HIV-negative $(24.5 \% \mathrm{v} .68 .3 \%)$, and the prevalence of gallstones as a cause was similar (23.6\% v. $17.9 \%)$. Antiretroviral therapy was associated with pancreatitis in $35.8 \%$, and $6(5.7 \%)$ had abdominal malignancies. Sixteen (15.1\%) had pancreatic necrosis, 20 (18.9\%) had septic complications, and 6 (5.7\%) died.

Conclusions. HIV-associated acute pancreatitis was most frequent in females and black Africans and was associated with malignancy. Mortality was similar in HIV and non-HIV pancreatitis.
\end{abstract}

S Afr Med J 2017;107(8):706-709. DOI:10.7196/SAMJ.2017.v107i8.10296

In environments with low HIV infection rates, acute pancreatitis is common in HIV-positive individuals. The causes of pancreatitis and the outcomes in this setting vary, ${ }^{[1]}$ with reports of acute pancreatitis being more prevalent and having poorer outcomes in HIV-infected patients than in patients without HIV infection. ${ }^{[1,2]}$ The studies in low-incidence settings were conducted during the early period of the use of highly active antiretroviral therapy (HAART) (the late 1990s) and the following decade, which saw the introduction of new agents and a decline in mortality associated with HIV infection. There is little information on the incidence and outcome of acute pancreatitis over an extended period during the HAART era.

\section{Objective}

To examine the prevalence and outcome of acute pancreatitis associated with HIV infection in a cohort of patients with acute pancreatitis admitted to a regional hospital service with a high prevalence of HIV infection during May 2001 - November 2010, a period that covers the introduction and expansion of HAART in the management of HIV infection in South Africa (SA).

\section{Methods}

All patients admitted to the adult surgical wards at Addington Hospital, Durban, SA, with a diagnosis of acute pancreatitis were included in a prospective dataset. Data from May 2001 to November 2010 were retrospectively analysed. The Glasgow criteria (score $\geq 3$ ) and organ dysfunction at presentation were used to assess prognosis. Complications related to acute pancreatitis and mortality were noted. HIV infection was sought by medical history. A diagnosis of acute pancreatitis was based on typical clinical symptoms (epigastric pain, nausea, vomiting) and a serum amylase level $\geq 3$ times the upper limit of normal (25 - $125 \mathrm{IU} / \mathrm{L})$ or a urinary amylase level $>760 \mathrm{IU} / \mathrm{L}$.
A contrast-enhanced computed tomography (CT) scan of the pancreas was performed in patients with predicted severe disease and in those with persistent symptoms at the end of the first week after admission. The CT scan was used to determine the presence of local complications of acute pancreatitis such as pancreatic necrosis, peripancreatic fluid collections and pancreatic ascites, and to confirm the diagnosis of pancreatitis when this was doubtful. The frequency of sepsis was analysed in HIV-positive and HIV-negative patients.

\section{Statistical analysis}

Descriptive statistics were used to determine the characteristics of the cohort. Continuous variables were expressed as means and ranges and categorical variables as percentages and proportions. Continuous variables were compared using the $t$-test and categorical variables using the $\chi^{2}$ test and Fisher's exact test. Statistical analysis was conducted using SPSS version 2.0 (IBM, USA). Results were considered statistically significant at $p<0.05$.

\section{Ethical approval}

Ethical approval was obtained from the local ethics committees of the Department of Health and the University of KwaZulu-Natal (ref. no. BE222/1).

\section{Results}

There were 627 admissions of patients with acute pancreatitis during the period May 2001 - November 2010, and 106 patients (16.9\%) were HIV-positive at presentation. The demographic details are set out in Table 1. Of the HIV-positive patients, most were female (65.1\%) and black African (92.5\%). The duration of symptoms ranged from 1 to 30 days, with a mean of 9.2 days. The CD4 count was known in 82 patients $(77.3 \%)$ with a mean of 220 cells $/ \mu \mathrm{L}$ (range 8 - 697); 
$51 / 82$ patients $(62.1 \%)$ had a CD 4 count $<200$ cells/ $\mu \mathrm{L}$. The body mass index was calculated in 81 patients, with a mean of $26 \mathrm{~kg} / \mathrm{m}^{2}$ (range 16 - 64).

The serum amylase level was used to confirm acute pancreatitis in 50 patients, with a mean of 1569 IU/L (range 375 - 5 769) and urinary amylase in 56 patients, with a mean of 4083 IU/L (range 934 - 36856 ). Of the $20 \mathrm{HIV}$-positive patients (18.9\%) with a serum amylase level $<1000 \mathrm{U} / \mathrm{L}, 7$ had evidence of acute pancreatitis on the CT scan. The prevalence of the common causes of pancreatitis differed between the HIV-positive and HIV-negative groups (Table 1). Alcohol was a less frequent cause in the HIV-positive group than in those who were HIVnegative $(p=0.0001)$, whereas the frequency of gallstones was similar ( $p=0.1741)$. Sixty-four $(60.4 \%)$ of the HIV-positive patients were on HAART (Table 2), and in 38 cases (59.4\%) this was the only possible cause of the pancreatitis. The Glasgow criteria predicted intensive care admission, complications and mortality with similar accuracy in the HIV-positive and HIV-negative patients. In 44 patients, imaging by CT scan $(n=23)$ and ultrasound $(n=21)$ showed a normal pancreas (Balthazar A), while in 46 patients, a CT scan $(n=39)$ and ultrasound $(n=7)$ showed features of acute pancreatitis.

In 13 HIV-positive patients (12.3\%), the CT scan and a raised erythrocyte sedimentation rate showed the pancreatitis to be associated with abdominal tuberculosis. Of the HIV-positive patients, 20 (18.9\%) had septic complications (6 chest infections, 5 central line sepsis, 3 septicaemia and 3 infections of necrotic pancreas). Two of the 3 patients with infected necrosis were successfully managed nonoperatively, and the other had a methicillin-resistant Staphylococcus aureus infection and died 2 weeks after admission. Eight patients presented with lactic acidosis, of whom 3 died within 1 week of admission. The rate of sepsis was significantly higher in the HIVpositive patients than in those who were HIV-negative (Table 1).

Six HIV-positive patients (5.7\%) had malignancies. These were four lymphomas in the head of the pancreas diagnosed by endoscopic ultrasound and aspiration, a pararenal lymphoma and a renal cell carcinoma diagnosed by CT scan and percutaneous aspiration. One of these patients died of disseminated disease at 2 weeks without features of pancreatic inflammation on a CT scan. No malignancies were found in the patients without HIV infection.

Four patients developed pseudocysts, which were managed expectantly. Six HIV-positive patients (5.7\%) died, of whom 3 (2 on HAART) had CD 4 counts $<200$ cells $/ \mu \mathrm{L}$.

\section{Discussion}

This and previous studies at Addington Hospital have demonstrated an increase in the prevalence of HIV in association with pancreatitis, from $5 \%(2001-2006)^{[3]}$ and $10 \%(2001-2008)^{[4]}$ to $16.9 \%$ in the present study (Fig. 1). An increasing incidence from 2.6 events per 1000 person-years for the period 1996 - 2000 to 5.1 events per 1000 personyears for $2001-2006$ ( $p=0.0014$ ) was reported by another group. ${ }^{[5]}$ The increase in the prevalence of HIV infection in our study was associated with an increase in gallstone-related pancreatitis (Fig. 1).

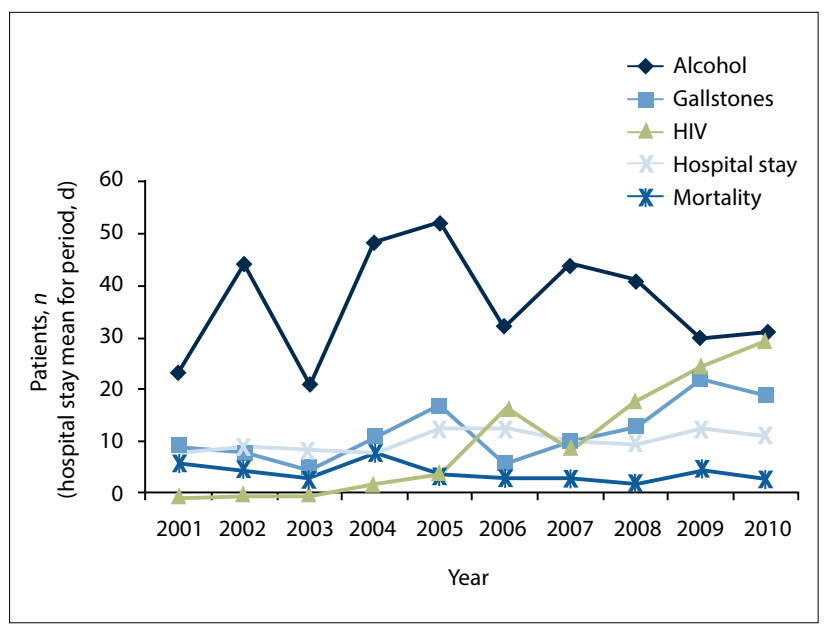

Fig. 1. Trends in aetiology, hospital stay and mortality in all patients with pancreatitis $(\mathrm{N}=627)$

Table 1. Characteristics of HIV-positive and HIV-negative patients $(N=627)$

\begin{tabular}{|c|c|c|c|}
\hline & HIV-positive & HIV-negative & $p$-value \\
\hline Total patients, $N(\%)$ & $106(16.9)$ & $521(83.1)$ & \\
\hline Males/females, $n(\%)$ & $37(34.9): 69(65.1)$ & $383(73.6): 138(26.5)$ & 0.000 \\
\hline Age (yr), mean (range) & $36(12-60)$ & $40(12-89)$ & 0.005 \\
\hline Duration of symptoms (d), mean (range) & $9.2(1-30)$ & $2(1-14)$ & 0.000 \\
\hline Body mass index $\left(\mathrm{kg} / \mathrm{m}^{2}\right)$, mean (range) & $26(16-64)$ & $25.4(16-59)$ & 0.76 \\
\hline \multicolumn{4}{|l|}{ Aetiology, $n(\%)$} \\
\hline Alcohol & $26(24.5)$ & $356(68.3)$ & 0.0001 \\
\hline Gallstones & $25(23.6)$ & $93(17.9)$ & 0.1741 \\
\hline Malignancy & $6(5.7)$ & 0 & 0.0001 \\
\hline \multicolumn{4}{|l|}{ Ethnic group, $n(\%)$} \\
\hline Black African & $97(91.5)$ & $226(43.4)$ & 0.000 \\
\hline Mixed race & $3(2.5)$ & $41(7.9)$ & 0.091 \\
\hline Indian & $6(5.7)$ & $232(44.6)$ & 0.0001 \\
\hline White & 0 & $22(4.2)$ & 0.036 \\
\hline \multicolumn{4}{|l|}{ Outcomes } \\
\hline Glasgow $\geq 3, n(\%)$ & $19(17.9)$ & $98(18.8)$ & 1.00 \\
\hline Organ failure, $n(\%)$ & $10(9.4)$ & $51(9.8)$ & 0.961 \\
\hline Hospital stay (d), mean (range) & $11.5(1-44)$ & $10.2(1-123)$ & 0.002 \\
\hline Necrosis pancreas, $n(\%)$ & $16(15.1)$ & $47(9.0)$ & 0.074 \\
\hline Sepsis, $n(\%)$ & $20(18.9)$ & $50(9.6)$ & 0.009 \\
\hline Complications, $n(\%)$ & $19(17.9)$ & $108(20.7)$ & 0.54 \\
\hline Mortality, $n(\%)$ & $6(5.7)$ & $37(7.1)$ & 0.611 \\
\hline
\end{tabular}


Table 2. Highly active antiretroviral therapy regimens $(N=64)$

\begin{tabular}{ll}
\hline & Patients, $\boldsymbol{n}$ \\
\hline Stavudine (NRTI)/lamivudine (NRTI)/efavirenz (NNRTI) & 56 \\
Stavudine (NRTI)/lamivudine (NRTI)/nevirapine (NNRTI) & 4 \\
Zidovudine (NRTI)/lamivudine (NRTI)/efavirenz (NNRTI) & 1 \\
Stavudine (NRTI)/nevirapine (NNRTI)/didanosine (NRTI) & 1 \\
Lamivudine (NRTI)/efavirenz (NNRTI)/tenofovir (PI) & 1 \\
Lamivudine (NRTI)/didanosine (NRTI)/nevirapine (NNRTI) & 1 \\
NRTI = nucleoside reverse transcriptase inhibitor; NNRTI = non-nucleoside reverse transcriptase inhibitor; PI = protease inhibitor. &
\end{tabular}

The majority of our patients with HIV-related pancreatitis were black African and female. Another study found more males (51.8\%) and more African Americans (88.2\%) with HIV-related pancreatitis. ${ }^{[5]}$ The mean ages of 35 and 38 years for females and males, respectively, is comparable to the $36,36.1$ and 37.4 years reported in previous studies. ${ }^{[5-7]}$ In a previous report, HIV-positive patients with acute pancreatitis were of similar age to those without pancreatitis. ${ }^{[6]}$

The association of lower CD4 counts with pancreatitis is unclear. In a previous study, there was a significant negative correlation between serum pancreatic enzyme levels and the number of CD4 lymphocytes ${ }^{[8]}$ A higher baseline CD4 cell count was associated with a decreased risk of pancreatitis. ${ }^{[9]}$ In our study, 51/82 patients (62.1\%) had CD4 counts $<200$ cells $/ \mu \mathrm{L}$, whereas in other studies the CD4 counts were $<200$ cells $/ \mu \mathrm{L}$ in $57.5 \%,{ }^{[5]} 32.6 \%,{ }^{[6]} 63 \%{ }^{[7]}$ and $89 \%$ of patients. ${ }^{[10]}$ In a previous report, ${ }^{[8]}$ it was also demonstrated that patients with asymptomatic HIV infection or a CD4 cell count $>500$ cells $/ \mu \mathrm{L}$ did not develop asymptomatic hyperamylasaemia or acute pancreatitis over a 1-year period.

The aetiology of acute pancreatitis in the setting of HIV infection also varies, and may include both causes common in HIV-negative individuals and HIV-specific causes that include medications, opportunistic infections and tumours. ${ }^{[1]}$ There is also a report of acute pancreatitis during primary HIV infection. ${ }^{[11]}$ In a previous study, pancreatitis was associated with gallstones, intravenous drug abuse, pentamidine intake, and Pneumocystis jiroveci and Mycobacterium avium-intracellulare infections. ${ }^{[8]}$ Acute pancreatitis is also a complication of nucleoside reverse transcriptase inhibitors (didanosine, stavudine). The combination of didanosine and stavudine was associated with the highest risk. ${ }^{[12]}$ In another study, the crude incidence of pancreatitis ranged from 0.18 cases per 100 person-years on therapy with zidovudine to 6.25 cases per 100 person-years for didanosine plus hydroxyurea. ${ }^{[13]}$ In a further report, there was no evidence of an association of pancreatitis with exposure to didanosine, stavudine or any other HAART regimen. ${ }^{[9]}$ There was a similar finding in another report without an association with a particular drug regimen. ${ }^{[14]}$ The HAART medications used by the patients in this study are listed in Table 2 .

A postmortem study of the pancreases of asymptomatic individuals with AIDS and normal antemortem ultrasound scans of the pancreas and normal serum amylase levels revealed mycobacteriosis (22\%), toxoplasmosis (13\%), cytomegalovirus (9\%), P. jiroveci (9\%) and HIV p24 (22\%) antigens in macrophage cytoplasm in the pancreas. ${ }^{[15]}$ Opportunistic infections of the pancreas have been reported in postmortem studies, but there is little information on the prevalence or diagnostic modalities appropriate for the antemortem environment. No patient in our cohort was diagnosed with opportunistic infections before death, and postmortem assessments were not performed. HAART, tuberculosis and malignancy were more frequent in HIV-positive patients (Table 3). In a previous report, drugs, alcohol, hypertriglyceridaemia and opportunistic
Table 3. Cause of pancreatitis and spectrum of $\mathrm{CD} 4$ counts and tumours in HIV-infected patients $(N=106)$

\begin{tabular}{ll}
\hline & $\boldsymbol{n}(\%)$ \\
\hline Cause of pancreatitis & \\
Alcohol & $26(24.5)$ \\
Gallstones & $25(23.6)$ \\
HAART & $38(35.8)$ \\
HIV only & $11(10.4)$ \\
CD4 count $($ cells $/ \mu \mathrm{L})(N=82)$ & \\
$\geq 200$ & $31(38.3)$ \\
$<200$ & $51(62.1)$ \\
Malignancy & \\
Lymphoma head of pancreas & $4(3.8)$ \\
Pararenal lymphoma & $1(0.9)$ \\
Renal cell carcinoma & $1(0.9)$ \\
HAART = highly active antiretroviral therapy. &
\end{tabular}

infections were the most common aetiological associations, ${ }^{[6]}$ and in another, $46 \%$ of cases were associated with medication and $26 \%$ had an idiopathic aetiology. ${ }^{[7]}$

Results reported on the accuracy of severity assessment indices are conflicting. In two studies, the Ranson and Glasgow criteria were found to be of poor prognostic value and the Apache II score a better predictor of an unfavourable outcome. ${ }^{[2,7]}$ However, the best overall accuracy of the Apache II system as a predictive tool (75\%) leaves a lot to be desired. ${ }^{[7]}$ In sharp contrast, another report demonstrated Ranson's criteria to be of similar value in predicting a poor outcome as was found in other non-HIV-related series. ${ }^{[16]}$ Lack of consensus on severity indices is added to by Parithivel et al.' $\mathrm{s}^{[10]}$ report, in which $89 \%$ of patients had CD 4 counts $<200$ cells/ $\mu \mathrm{L}$. They found that the commonly used predictors of severe disease were unhelpful because of altered hepatic and renal function and low white cell counts in HIV-infected patients. In our study, the frequencies of a Glasgow score of $\geq 3$, organ failure and mortality were found to be similar in the HIV-positive and HIV-negative groups.

The mortality rate of $5.7 \%$ in this study is similar to the rate of $5.9 \%$ reported by Smith et al. ${ }^{[9]}$ but is much lower than the $32 \%$ reported a decade earlier in a study that had a significant number of patients with AIDS in the cohort. ${ }^{[10]}$

\section{Conclusions}

In our setting, acute pancreatitis associated with HIV infection was most common in females and in black African patients. The serum amylase level was elevated for prolonged periods and may be used to aid diagnosis after 72 hours of symptoms. Drug-related pancreatitis was more frequent and alcohol as a cause less frequent in HIVrelated pancreatitis. Malignancy in this cohort was associated with HIV infection. Although pancreatic necrosis was more common in HIV-related pancreatitis, mortality was similar. However, it was 
not possible to draw firm conclusions from this cohort, as not all patients admitted with pancreatitis were tested for HIV infection and there may have been a bias towards those with more advanced HIV infection. A prospective study with routine testing of all admissions with acute pancreatitis is required to ascertain whether these trends are applicable to all with HIV-related pancreatitis.

Acknowledgements. This article was presented at the International Hepato-Pancreato-Biliary Association meeting in Paris on 1 - 5 July 2012, at which it was submitted for best oral presentation.

Author contributions. FA compiled the Excel worksheet, analysed the data and completed the article with revisions. SRT was involved in the conception of the research and the initial writing of the article.

Funding. None.

\section{Conflicts of interest. None.}

1. Dassopoulos T, Ehrenpreis ED. Acute pancreatitis in human immunodeficiency virus-infected patients: A review. Am J Med 1999;107(1):78-84. https://doi.org/10.1016/S0002-9343(99)00169-2

2. Cappell MS, Marks M. Acute pancreatitis in HIV-seropositive patients: A case control study of 44 2. Cappell MS, Marks M. Acute pancreatitis in HIV-seropositive patients: A case control s
patients. Am J Med 1995;98(3):243-248. https://doi.org/10.1016/S0002-9343(99)80370-2

3. Anderson F, Thomson S, Clarke D, Loots E. Acute pancreatitis: Demographics, aetiological factors and outcomes in a regional hospital in South Africa. S Afr J Surg 2009;46(3):83-86.

4. Anderson F, Mbatha SZ, Thomson SR. The early management of pancreatitis associated with hypertriglyceridaemia. S Afr J Surg 2011;49(2):82-84
5. Riedel DJ, Gebo KA, Moore RD, Lucas GM. A ten-year analysis of the incidence and risk factors for acute pancreatitis requiring hospitalization in an urban HIV clinical cohort. AIDS Patient Care STDS 2008;22(2):113-121. https://doi.org/10.1089/apc.2007.0034

6. Manfredi R, Calza L. HIV infection and the pancreas: Risk factors and potential management guidelines. Int J STD AIDS 2008;19(2):99-105. https://doi.org/10.1258/ijsa.2007.007076
gantere

7. Gan I, May G, Raboud J, Tilley J, Enns R. Pancreatitis in HIV infection: Predictors of severity. Am J

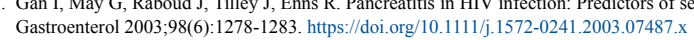

8. Dutta S, Ting C, Lai L. Study of prevalence, severity, and etiological factors associated with acute pancreatitis in patients infected with human immunodeficiency virus. Am J Gastroenterol 1997;92(11):2044-2048

9. Smith CJ, Olsen $\mathrm{CH}$, Mocroft A, et al. The role of antiretroviral therapy in the incidence of pancreatitis in HIV-positive individuals in the EuroSIDA study. AIDS 2008;22(1):47-56. https://doi.org/10.1097/ QAD.0b013e3282f03094

10. Parithivel VS, Yousuf AM, Albu E, Kaul A, Aydinalp N. Predictors of the severity of acute pancreatitis in patients with HIV infection or AIDS. Pancreas 1999;19(2):133-136.

11. Mortier E, Gaba S, Mari I, Vinceneux P, Pouchot J. Acute pancreatitis during primary HIV-1 infection. Am J Gastroenterol 2002;97(2):504-507. https://doi.org/10.1111/j.1572-0241.2002.05519.x

12. Dragovic G, Milic N, Jevtovic D. Incidence of acute pancreatitis and nucleoside reverse transcriptase inhibitors usage. Int J STD AIDS 2005;16(6):427-429.

13. Moore RD, Keruly JC, Chaisson RE. Incidence of pancreatitis in HIV-infected patients receiving nucleoside reverse transcriptase inhibitor drugs. AIDS 2001;15(5):617-620.

14. Guo JJ, Jang R, Louder A, Cluxton RJ. Acute pancreatitis associated with different combination Guo JJ, Jang R, Louder A, Cluxton RJ. Acute pancreatitis associated with different combination
therapies in patients infected with human immunodeficiency virus. Pharmacotherapy 2005;25(8):1044therapies in patients infected with human immunode
1054. https://doi.org/10.1592/phco.2005.25.8.1044

15. Chehter EZ, Longo MA, Laudanna AA, Duarte MIS. Involvement of the pancreas in AIDS: A prospective study of 109 post-mortems. AIDS 2000;14(13):1879-1886.

16. Manocha AP, Sossenheimer M, Martin SP, et al. Prevalence and predictors of severe acute pancreatitis in patients with acquired immune deficiency syndrome (AIDS). Am J Gastroenterol 1999;94(3):784789. https://doi.org/10.1111/j.1572-0241.1999.00951.x

Accepted 11 May 2017. 